\title{
Phytoplancton en baie de Seine. Influence du panache fluvial sur la production primaire
}

\author{
Christiane VIDEAU ${ }^{\text {a }}$, Mireille RYCKAERT ${ }^{\text {b }}$, Stéphane L'HELGUEN ${ }^{\text {a }}$ \\ ${ }^{a}$ Laboratoire d'océanographie chimique, UPR 9042 CNRS Roscoff \\ et université de Bretagne occidentale, 4, place Copernic, Technopôle Brest-Iroise, 29280 Plouzané, France \\ ${ }^{\mathrm{b}}$ Département environnement littoral, Ifremer, place du Séminaire, BP 7, 17137 L'Houmeau, France
}

(Revisé le 26 mars 1998, accepté le 3 avril 1998)

\begin{abstract}
Phytoplankton in the Bay of Seine (France). Influence of the river plume on primary productivity. Phytoplankton distribution and primary productivity were investigated in the Bay of Seine (eastern English Channel, France) in spring and early summer of 1992 and 1994. In 1992, the horizontal distribution of phytoplankton species was determined over the whole Bay of Seine. In 1994, species distribution and primary productivity were studied along the salinity gradient of the Seine plume and in the neighbouring marine waters. Phytoplankton distribution was characterised by the permanent diatom dominance from early spring to early summer. The spring bloom did not occur uniformly over the bay, but was initiated in the marine waters, in the middle of the bay and progressed towards the coast where it developed, in June, in the diluted waters of the Seine plume. Phytoplankton distribution was also characterised by the dominance of large diatoms (Rhizosolenia) in marine waters and by the dominance of small species (Skeletonema costatum, Asterionella glacialis) in the plume waters. The greatest abundance of large diatoms was generally encountered below the euphotic zone. This accumulation seemed to be related to cell sedimentation and advection of marine waters below the diluted plume waters. The daily carbon productivity range was 1.4 to $3 \mathrm{~g} \mathrm{~m}^{-2} \mathrm{~d}^{-1}$. The highest values were measured in the plume waters $(S>28)$, suggesting the primary productivity in the plume was more controlled by light than by nutrients. The photic zone productivity/ chlorophyll $a$ ratios ( $P / B$; producted carbon per unit of Chlat were close $1040 \mathrm{mg} \mathrm{mg}^{-1} \mathrm{~d}^{-1}$ in the plume waters. $\mathrm{P} / \mathrm{B}$ exceptionally reached $90 \mathrm{mg} \mathrm{mg}^{-1} \mathrm{~d}^{-1}$ in early summer. This value corresponded to a fast growing population of $S$, costatum. In marine waters, $P / B$ varied from $22 \mathrm{mg} \mathrm{mg}^{-1} \mathrm{~d}^{-1}$, in spring, to $43 \mathrm{mg} \mathrm{mg}^{-1} \mathrm{~d}^{-1}$, in early summer. (O) Elsevier, Paris
\end{abstract}

\section{phytoplankton / primary productivity / Bay of Seine}

Résumé - La distribution des populations phytoplanctoniques et la production primaire ont été étudiées, au printemps et au début de l'été, en 1992 sur l'ensemble de la baie de Seine et en 1994, dans le gradient de salinité du panache de la Seine et dans les eaux marines adjacentes. La distribution du phytoplancton est marquée par la dominance persistante des diatomées du début du printemps au début de l'été. Leur développement n'est pas uniforme. La floraison printanière des diatomées débute dans les eaux marines, au centre de la baie et progresse vers la côte où elle se développe, en juin, dans les eaux dessalées. La distribution du phytoplancton est aussi caractérisée par la prédominance des diatomées de grande taille (Rhizosolenia) dans les eaux marines et par celle des espèces de petite taille (Skeletonema costatum. Asterionella glacialis) dans les eaux du panache. Les densités maximales des diatomées de grande taille sont généralement observées en dessous de la couche éclairée ; ces accumulations semblent liées, d'une part, à la sédimentation des cellules et, d'autre part, à l'advection des eaux marines sous les eaux dessalées du panache. Les valeurs de production journalière de carbone varient de 1,4 à $3 \mathrm{~g} \mathrm{~m}^{-2} \mathrm{j}^{-1}$ : les valeurs les plus élevées sont mesurées dans le panache à des salinités supérieures à 28 ce qui sug- 
gère que la production phytoplanctonique est davantage contrôlée par la lumière que par les éléments nutritifs. La productivité des masses d'eau $\mathrm{P} / \mathrm{B}$ (carbone produit par unité de Chla) est voisine de $40 \mathrm{mg} \mathrm{mg}^{-1} \mathrm{j}^{-1}$ dans les eaux du panache. Elle atteint exceptionnellement $90 \mathrm{mg} \mathrm{mg}^{-1} \mathrm{j}^{-1}$, avec la forte croissance d'une population de $S$. costatum. Dans les eaux marines, $\mathrm{P} / \mathrm{B}$ varie de $22 \mathrm{mg} \mathrm{mg}^{-1} \mathrm{j}^{-1}$ au printemps à $43 \mathrm{mg} \mathrm{mg}^{-1} \mathrm{j}^{-1}$ au début de l'été. () Elsevier, Paris

\section{phytoplancton / production primaire / baie de Seine}

\section{INTRODUCTION}

Les grands fleuves déversent dans les eaux côtières des quantités considérables d'éléments nutritifs. L'importance de cet enrichissement dépend, à la fois de l'étendue des bassins versants et de l'intensité des activités domestiques, agricoles et industrielles qui s*y développent. Cette eutrophisation peut modifier fortement le fonctionnement des systèmes côtiers. Elle peut, en particulier, engendrer une augmentation de la production phytoplanctonique. La dégradation et la minéralisation de la matière organique ainsi produite peuvent aboutir à un appauvrissement en oxygène dissous des masses d'eau. susceptible d'entraîner une forte mortalité de la faune marine.

Les eaux de la baie de Seine (Manche orientale) sont un exemple d'eaux côtières soumises à l'influence d'importants apports continentaux. Les apports d'eau douce, en raison du fort débit de la Seine $\left(400 \mathrm{~m}^{3} \mathrm{~s}^{-1}\right.$ en moyenne interannuelle [1]) sont très importants et induisent la formation d'un panache dont le sens d'écoulement, vers l'ouest et le nord-ouest [2] est mis en évidence par la turbidité des eaux superficielles qui évolue selon un gradient proche du gradient de salinité. Les eaux dessalées s'écoulent en surface et non sur toute la colonne d'eau malgré l'intensité des courants de marée ; il en résulte un gradient vertical de salinité permanent dans la partie orientale de la baie de Seine [9]. Les eaux dessalées du panache véhiculent des quantités importantes d'éléments nutritifs. Les flux d'éléments nutritifs déversés par la Seine sont dépendants du débit du fleuve pour l'azote et les silicates et indépendants du débit pour les phosphates [28]. Aminot et al. [1] estiment la quantité d'azote minéral déversée en baie de Seine à $80000 \mathrm{t} \mathrm{a}^{-1}$ pour une année sèche (1992) et à $130000 \mathrm{t} \mathrm{a}^{-1}$ pour une année humide (1994). lls évaluent les apports de silicates à 40000 et $77000 \mathrm{t} \mathrm{a}^{-1}$ respectivement, et les apports en phosphates à 8400 à $6500 \mathrm{t} \mathrm{a}^{-1}$.

La baie de Seine est fréquemment le siège de développements phytoplanctoniques importants $\left(20 \mathrm{à} 30 \mathrm{\mu g} \mathrm{L}^{-1}\right.$ Chla) du début du printemps à la fin de la période estivale
[14]. Les fortes biomasses chlorophylliennes observées correspondent principalement au développement de diatomées (70 à $90 \%$ de la densité cellulaire du microphytoplancton), représentées essentiellement par les genres Chaetoceros, Rhizosolenia et Thalassiosira. Dans le panache de la Seine, on observe périodiquement de véritables phénomènes d'eaux colorées où la teneur en chlorophylle peut atteindre $100 \mu \mathrm{g} \mathrm{L}^{-1}$ [30]. Les organismes responsables de la coloration de l'eau sont des diatomées (genres précédemment cités) accompagnées ou non de dinoflagellés (Dinophysis, par exemple), de phytoflagellés (Phaeocystis), de ciliés (Mesodinium rubrum), ou de silicoflagellés (Dichtyochta).

Malgré ces observations, les relations entre les apports de la Seine et l'apparition des fortes biomasses phytoplanctoniques demeurent relativement peu connues. Deux séries de campagnes à la mer ont été effectuées afin d'examiner l'influence des apports en éléments nutritifs de la Seine sur la production primaire phytoplanctonique. Au cours des campagnes Phytoseine/Sediseine (1992), la distribution du phytoplancton a été examinée sur l'ensemble de la baie de Seine. Les campagnes Nutriseine (1994) ont été consacrées plus particulièrement au panache de la Seine; la production phytoplanctonique a été quantifiée dans les différents secteurs du panache et dans les eaux marines adjacentes.

\section{MATÉRIEL ET MÉTHODES}

\section{1. Échantillonnage}

\subsection{Campagnes Phytoseine/Sediseine (1992)}

Trois campagnes (N.O. Thalia) ont été effectuées en 1992 (Phytoseine 1, 24-30 avril, Sediseine, 2-4 juin et Phytoseine 2,29 juin-3 juillet). Les stations de prélèvement sont réparties sur l'ensemble de la baie (figure 1). Les prélèvements ont été effectués en surface à l'aide de bouteilles Niskin. La composition floristique a été déterminée sur chacun des échantillons. 


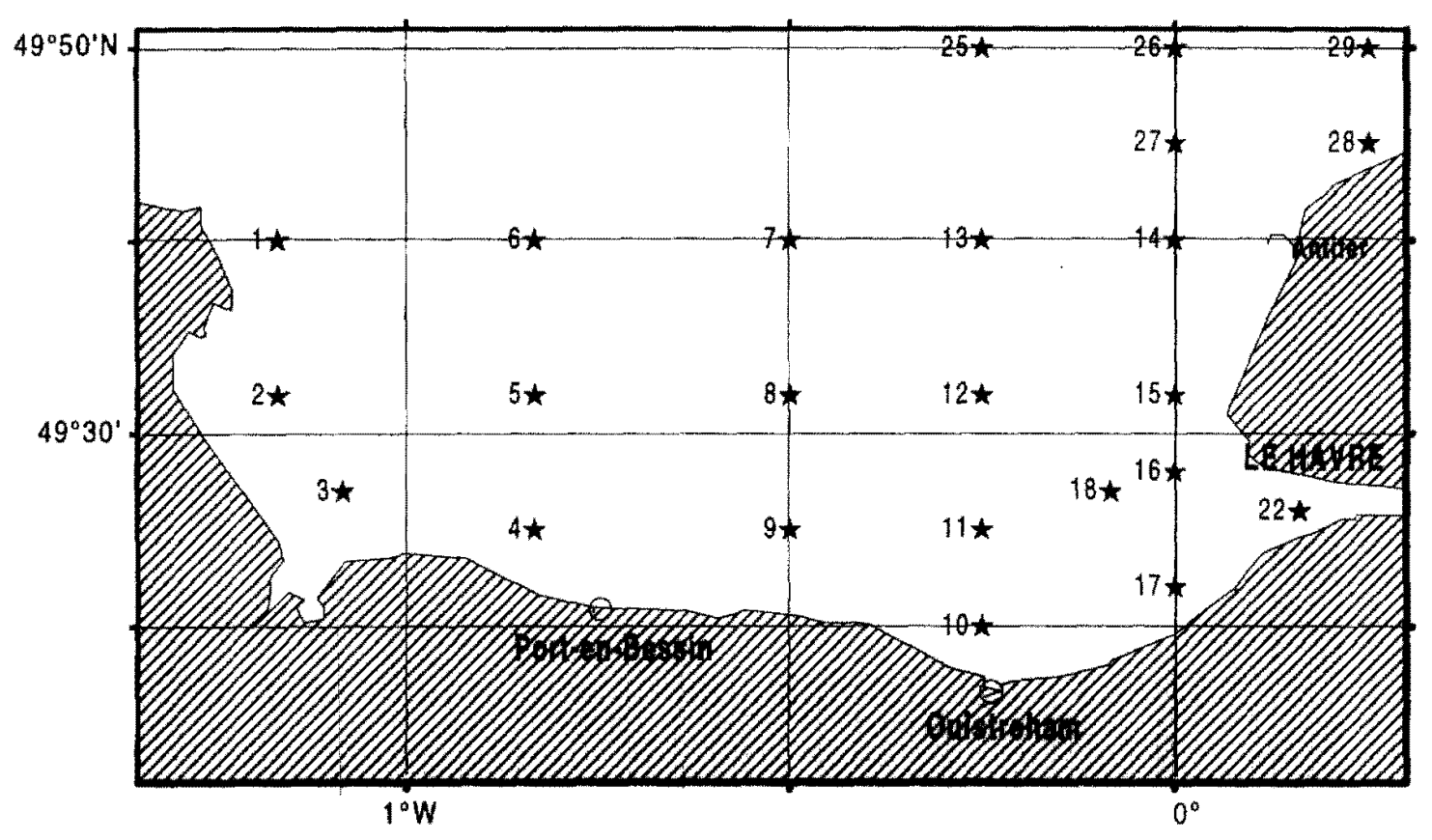

Figure 1. Position des points de prélèvement lors des campagnes Phytoseine/Sediseine (1992).

Figure 1. Sampling location during Phytoseine/Sediseine cruises (1992).

\subsubsection{Campagnes Nutriseine (1994)}

Les campagnes Nutriseine (N.O. Thalia) correspondent à des régimes de la Seine et à des phases de développement phytoplanctonique différents. Nutriseine I (25-29 avril) a été réalisée à la fin de période de crue et en période de floraison printanière et Nutriseine 2(22-26 juin) au début de la période d'étiage. Elles ont été programmées en périodes de vives-eaux.

Au cours de chaque campagne, quatre types de masses d'eau sont examinés. Les points de prélèvement (figure 2) sont définis par la salinité de surface de façon à distinguer un point proximal (point $D, S=25$ ), un point médian, (point $C, S=28$ ) et un point distal (point $B, S=32$ ) du panache ainsi qu'un point marin (point $A, S>34$ ). A chaque station, les profils de température, de salinité, de fluorescence et de photométrie sont obtenus en temps réel grâce à une sonde CTD Seabird modèle SBE 19 Seacat Profiler, couplée à un fluoromètre Sea Tech 500 et un photomètre Licor II-193 SA PAR. Les prélèvements d'eau de mer sont effectués à l'aide de bouteilles Niskin de $30 \mathrm{~L}$; trois à quatre niveaux sont échantillonnés dans la couche éclairée et un dans la couche sous-jacente. La salinité, les concentrations en éléments nutritifs, les concentrations en chlorophylle $a$ et la composition floristique sont déterminées sur chacun des échantillons. L'assimilation du carbone est mesurée également à chaque profondeur; le taux de croissance spécifique est déterminé en surface et à la profondeur correspondant à $10 \%$ de l'éclairement incident de surface. Les résultats sont présentés sur une coupe fictive qui ne tient pas compte des distances entre les stations.

\subsection{Méthodes d'analyse}

Les mesures de salinité sont effectuées à l'aide d'un salinomètre à électrodes Guidline Autosal modèle 8400. Les nitrates, nitrites, phosphates et silicates sont dosés en analyse automatique sur un Autoanalyzer II Technicon, selon la procédure de Tréguer et Le Corre [39]. Le dosage de l'ammonium est effectué manuellement par la méthode colorimétrique de Koroleff [18].

Les pigments chlorophylliens sont extraits dans l'acétone à $90 \%$ et dosés selon la méthode de Yentsch et Menzel [44] sur un fluoromètre Turner modèle III avant et après acidification $(\mathrm{HCl}, 1 \mathrm{~N})$. Les concentrations en chlorophylle $a$ et phéophytine $a$ sont calculées selon les équations de Lorenzen [22]. 


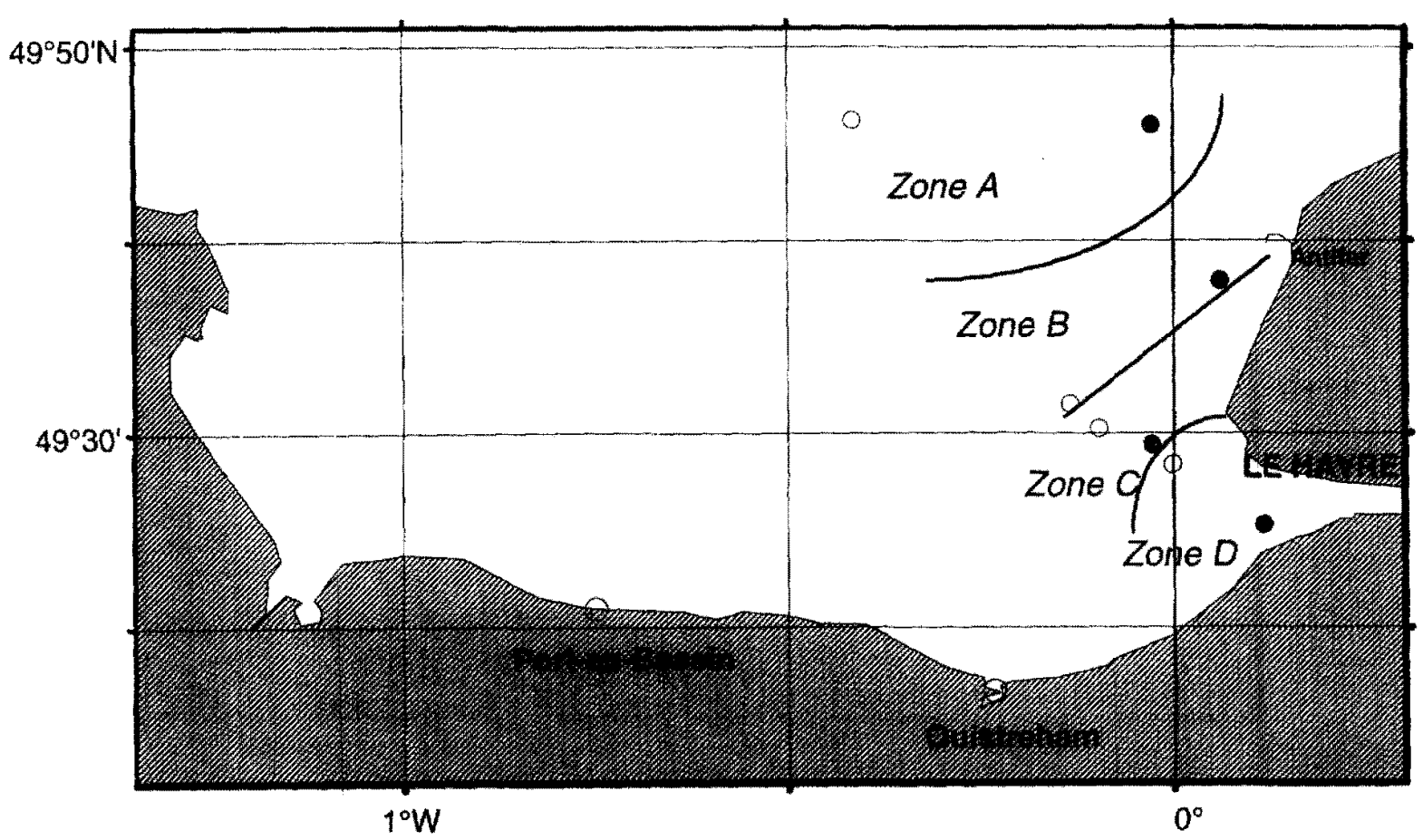

Figure 2. Position des stations de prélèvement lors des campagnes Nutriseine (1994), en avril (cercles clairs), juin (cercles noirs). Zone A : eaux marines $(S>34)$; Zone $B:$ zone distale du panache $(S=32) ;$ Zone $C:$ zone médiane du panache $(S=28-29) ; Z$ one $D: z o n e$ proximale du panache $(S=24-25$ ).

Figure 2. Sampling location during Nutriseine cruises (1994) in April (open circles), June (black circles). Zone A: marine waters (S > 34); Zone $B$ : apex of the Seine plume $(S=32)$; Zone $C$ : middle plume $(S=28-29)$; Zone D: upper plume $(S=24-25)$.

Pour la détermination de la composition floristique, les échantillons d'eau de mer sont fixés immédiatement après le prélèvement avec une solution de Lugol acide. Les comptages sont effectués après sédimentation, selon la méthode d'Utermöhl [40], sur un microscope inversé Zeiss (Teraval 31 ).

Les échantillons destinés aux mesures des taux de production primaire sont préalablement filtrés sur une soie Nytex (maille de $200 \mu \mathrm{m}$ ). La production carbonée est estimée par la méthode au ${ }^{14} \mathrm{C}$ [37]. Le carbone, fourni sous forme de $\mathrm{NH}^{14} \mathrm{CO}_{3}$ (Amersham) est ajouté à raison de $0,18 \mathrm{MBq}$ dans chacun des échantillons (environ $120 \mathrm{~mL}$ ). Les incubations sont in situ simule, entre $10 \mathrm{~h}$ T.U et $14 \mathrm{~h}$ T.U. Les échantillons sont ensuite filtrés sur des membranes Millipore AA. L'incorporation du carbone est mesurée en scintillation liquide dans un liquide scintillant BCS (Packard) sur un compteur à scintillation Tricarb 1600 TR (Packard). Les taux horaires de fixation du carbone sont intégrés sur l'ensemble de la couche euphotique La production journalière, estimée en multi- pliant les valeurs intégrées par la durée du jour (13 h en avril et $16 \mathrm{~h}$ en juin) est probablement surestimée.

Le taux de croissance spécifique du phytoplancton $\mu$ est déterminé par incorporation de ${ }^{14} \mathrm{C}$ dans les molécules de chlorophylle [32] selon la méthode de Welschmeyer et Lorenzen [43]. Des échantillons de 2 à $4 \mathrm{~L}$ d'eau de mer (selon la biomasse chlorophyllienne) sont incubés in situ simulé du midi solaire au coucher du soleil, en présence de $9,25 \mathrm{MBq}$ de $\mathrm{Na} \mathrm{H}^{14} \mathrm{CO}_{3}$. Le protocole expérimental est identique au précédent $[41,42]$.

\section{RÉSULTATS}

\subsection{Campagnes Phytoseine/Sediseine}

En avril, les plus fortes densités de phytoplancton sont observées dans les eaux marines. Le microphytoplancton est composé príncipalement de diatomées ; leurs densités atteignent plus de $4 \times 10^{5}$ cellules par litre (figure $3 a$ ). 

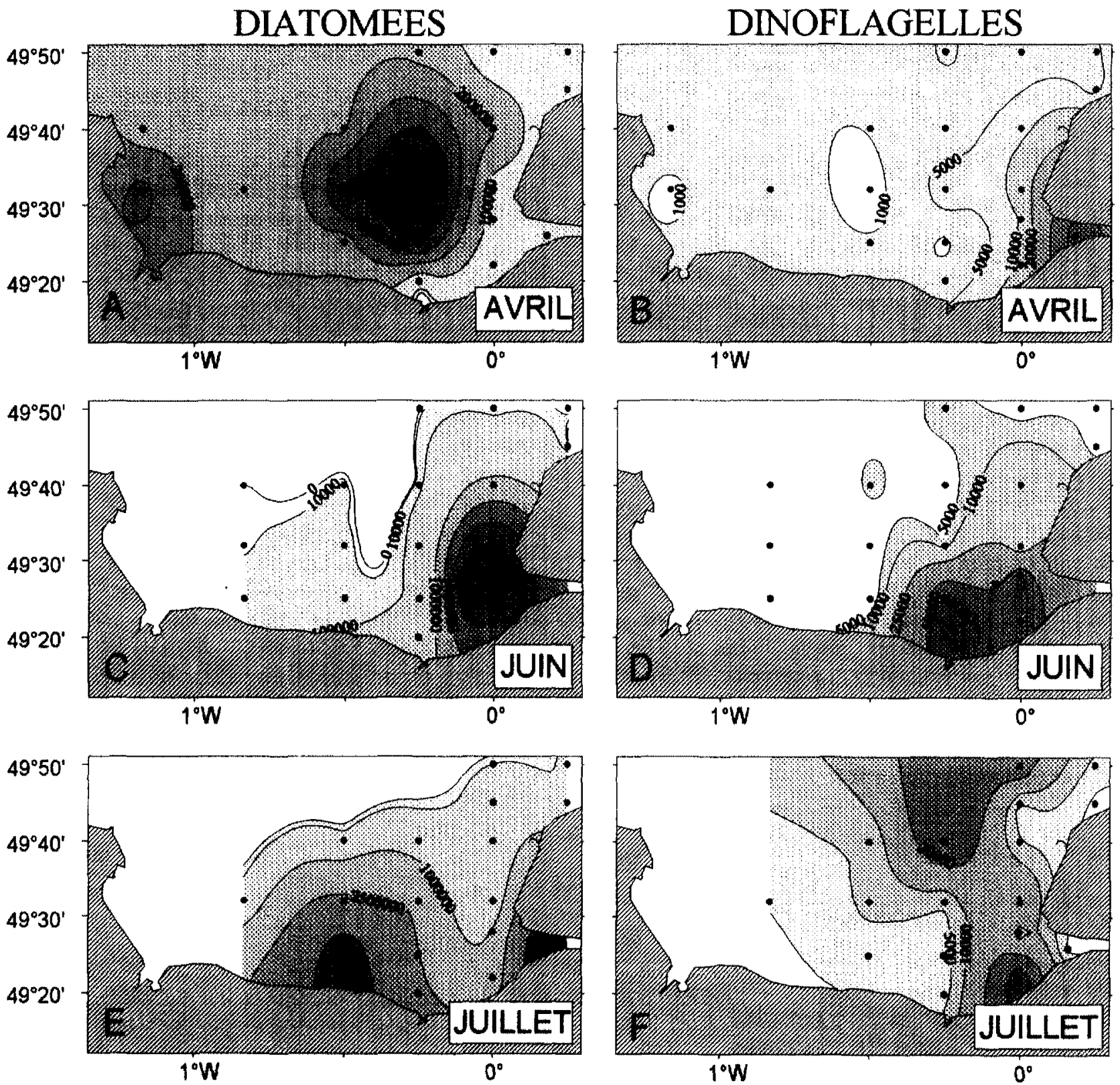

Figure 3. Distribution horizontale des deux principaux groupes de microphytoplancton (cellules $\mathrm{L}^{-1}$ ) en baie de Seine, en 1992 : A, B, avril, C, D, juin, E, F, juillet.

Figure 3. Horizontal distribution of the two main groups of microphytoplankton (cells $\mathrm{L}^{-1}$ ) in the Bay of Seine during 1992: A, B, April, C, D, June, E, F, July.

Les principales espèces recensées sont des espèces du genre Rhizosolenia (R.delicatula, $R$. schrubsolei, $R$. stolterfothii. par ordre décroissant d'abondance) ; elles représentent 60 à $80 \%$ du phytoplancton de taille supérieure à 10-15 $\mu \mathrm{m}$. Dans le panache, les diatomées sont peu abon- dantes $\left(<10^{5}\right.$ cellules par litre) ; elles sont représentées par de petites espèces telles que Chaetoceros socialis à proximité de l'estuaire de la Seine ou Asterionella glacialis, dans la partie sud de la baie. Dans les deux cas, la densité cellulaire de ces diatomées n'excède pas $10^{4}$ cel- 
lules par litre. II existe une bonne concordance entre les densités de diatomées et les concentrations en chlorophylle a [1]. Cependant, dans l'ouest et le nord de la baie, le phytoflagellé Phaeocystis dont la densité est voisine de $10^{7}$ cellules par litre contribue également à la biomasse chlorophyllienne. Les dinoflagellés, dont le nombre de cellules n'excède pas $6 \times 10^{3}$ par litre, sont localisés essentiellement dans le panache de la Seine (figure $3 b$ ).

A la fin de la période printanière et au début de l'été, les fortes concentrations de phytoplancton (diatomées) sont limitées aux eaux dessalées. Les populations dominantes sont constituées, en juin, d'Asterionella glacialis dont la plus forte abondance $\left(3 \times 10^{6}\right.$ cellules par litre) se rencontre à proximité de l'estuaire (jigure $3 c$ ) et en juillet, d'A. glacialis $\left(6 \times 10^{6}\right.$ cellules par litre) dans l'estuaire et de Chaetoceros socialis $\left(5 \times 10^{6}\right.$ cellules par litre), dans le sud de la baie (figure $3 e$ ). Dans cette zone, l'abondance des diatomées de petite taille diminue de la côte vers le large, au profit du genre Rhizosolenia ( $R$. delicatula, $R$. stolterfothii) dont la densité atteint 1 à $2 \times 10^{5}$ cellules par litre dans les eaux marines. Les dinoflagellés, représentés principalement par Prorocentrum minimum, demeurent peu abondants. Les plus fortes densités $(6 \times$ $10^{4}$ cellules par litre, en juin) sont observées au sud de l'estuaire de la Seine (figures $3 d, f$ ).

\subsection{Campagnes Nutriseine}

\subsubsection{Distribution de la salinité et de la température}

La distribution de la salinité (figures $4 a$ et $b$ ) met en évidence deux forts gradients, vertical et horizontal, qui soulignent l'extension du panache. Pour une salinité de surface donnée, la colonne d'eau présente une stratification, identique en avril et en juin, qui persiste jusqu'à une salinité superficielle de 33.

Les gradients thermiques sont moins prononcés que les gradients halins (tableau $\mathrm{D}$. La température de surface diminue, en avril de $10,8^{\circ} \mathrm{C}$ au point proximal à $10,1^{\circ} \mathrm{C}$ au point distal du panache, et en juin de $17,3{ }^{\circ} \mathrm{C}$ à $15,2^{\circ} \mathrm{C}$. Le gradient vertical de température est faible aux points proximal et distal (inférieur à $0,8^{\circ} \mathrm{C}$ ), légèrement plus élevé au point médian $\left(1,2^{\circ} \mathrm{C}\right.$ en avril et juin). Dans les eaux marines, la colonne d'eau est thermiquement homogène en avril $\left(9,1^{\circ} \mathrm{C}\right)$ et en juin $\left(13,6^{\circ} \mathrm{C}\right)$.

Le coefficient d'extinction de la lumière $\mathrm{k}$ (tableau $n$ ) diminue, en avril, de $0,99 \mathrm{~m}^{-1}$ à $0,31 \mathrm{~m}^{-1}$ du point proximal au point distal du panache et, en juin, de $1,44 \mathrm{~m}^{-1}$ à
$0,35 \mathrm{~m}^{-1}$. Dans les eaux marines, $\mathrm{k}$ varie de $0,18 \mathrm{~m}^{-1}$ en avril à $0,25 \mathrm{~m}^{-1}$ en juin.

\subsubsection{Distribution des concentrations en sels nutritifs}

La distribution verticale des teneurs en nitrates suit celle de la salinité avec un fort gradient horizontal et vertical (figures $4 c$ et $d$ ). Il en est de même pour l'ammonium, les silicates et les phosphates (non montre). En avril, les teneurs en nitrates dans les eaux du panache sont très élevées, jusqu'à $115 \mu \mathrm{mol} \mathrm{L} \mathrm{L}^{-1}$ au point proximal, en surface. Les concentrations de nitrates diminuent selon le gradient de salinité mais demeurent élevées jusqu'au point distal du panache $\left(19 \mu \mathrm{mol} \mathrm{L} \mathrm{L}^{-1}\right)$. Dans les eaux marines, elles sont nettement plus faibles ( 7 mol $\left.\mathrm{L}^{-1}\right)$. Les autres éléments nutritifs suivent une évolution identique (tableau $I$ ). Les concentrations d'ammonium diminuent, du point proximal au point distal du panache, de 6 à $0,7 \mu \mathrm{mol} \mathrm{L} \mathrm{L}^{-1}$, celles des silicates de 27 à $6,5 \mu \mathrm{mol} \mathrm{L}^{-1}$ et celles des phosphates de 2,5 à $1 \mu \mathrm{mol} \mathrm{L}^{-1}$. Dans les eaux marines, la concentration en ammonium est de $0,6 \mu \mathrm{mol} \mathrm{L} \mathrm{L}^{-1}$, celle des silicates de $0,7 \mu \mathrm{mol} \mathrm{L}^{-1}$ et celle des phosphates de $0,3 \mu$ mol $L^{-1}$. En juin, les teneurs en éléments nutritifs, pour des valeurs de salinité comparables, sont moins élevées qu'en avil. Les eaux du panache demeurent fortement enrichies à l'embouchure de l'estuaire. Les concentrations diminuent rapidement le long du gradient de salinité (tableau n). Dans les eaux marines, seules les concentrations en nitrates $\left(1,3 \mu \mathrm{mol} \mathrm{L^{-1 } )}\right.$ ont fortement chuté par rapport à avril.

\subsubsection{Distribution de la chlorophylle a et du phytoplancton}

En avril, la teneur en chlorophylle $a$ varie, dans le pana-

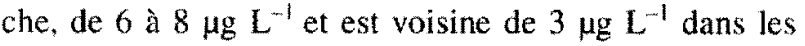
eaux marines (figure 4e). Le maximum de chlorophylle a, dans le panache, se situe le long de l'isohaline $S=31$; au point proximal, il se trouve en dessous de la zone euphotique et au point médian à la profondeur correspondant à $10 \%$ de l'éclairement de surface. Le microphytoplancton est composé essentiellement de diatomées. L'espèce Rhizosolenia delicatula est dominante aux différents points du panache et dans les eaux marines. Cette espèce représente, en surface, environ $60 \%$ du nombre total des diatomées, proportion qui augmente avec la profondeur pour atteindre 70 à $75 \%$ au-dessous de la zone euphotique. Le gradient vertical de la densité cellulaire de $R$. delicatula est particulièrement marqué au point proxi- 

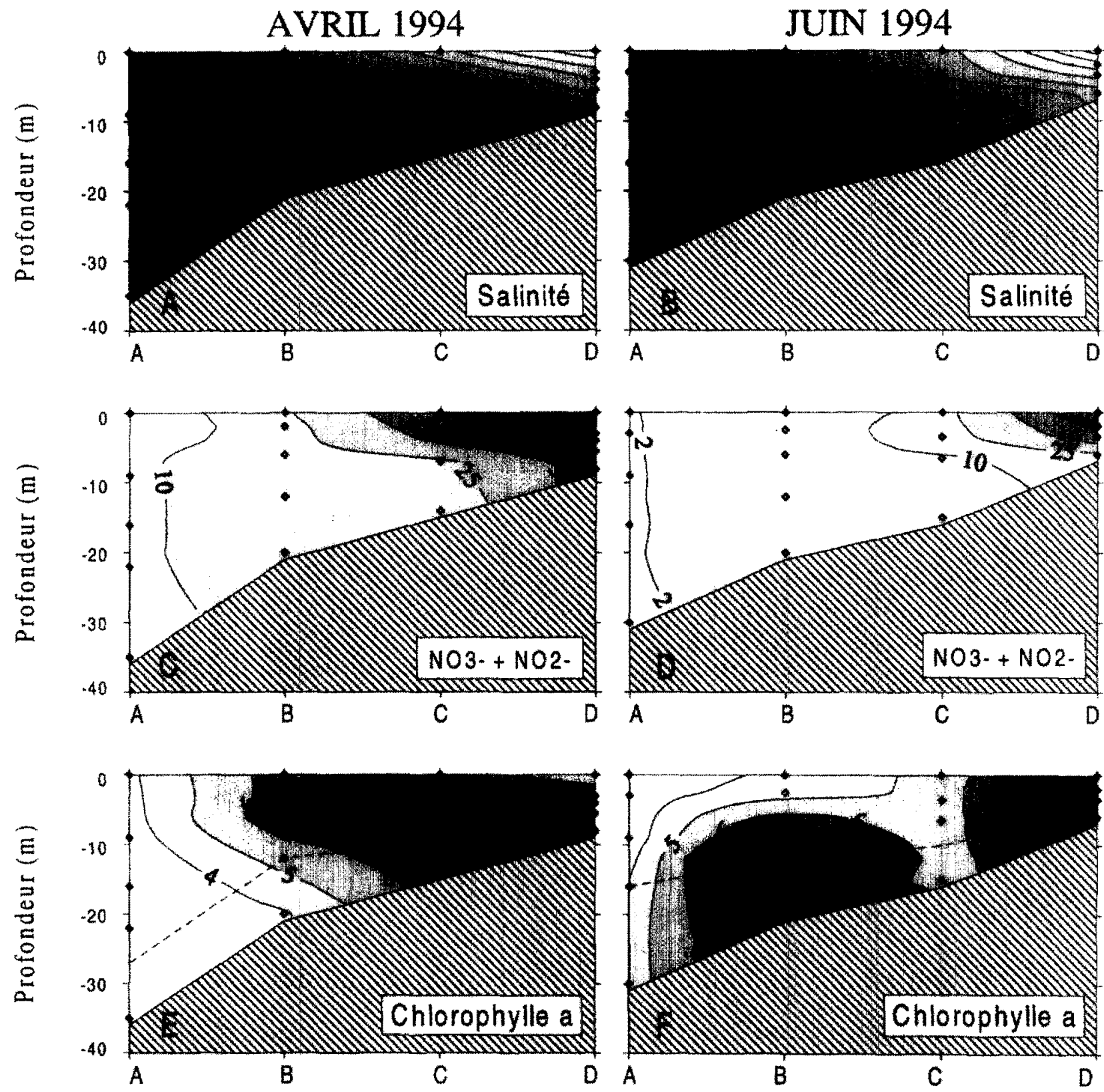

Figure 4. Distribution verticale: $A, B$ : de la salinité $; C, D:$ des nitrates $\left(\mu m o l L^{-1} \mathrm{~N}-\left(\mathrm{NO}_{3}+\mathrm{NO}_{2}\right)\right.$ ) $\mathrm{E}, \mathrm{F}:$ de la chlorophylle a ( $\left.\mu \mathrm{g} \mathrm{L}^{-1}\right)$ en avril et en juin 1994. Les points correspondent aux niveaux de prélèvement; la ligne pointillée représente la limite de la zone euphotique.

Figure 4. Vertical distribution: A, B: salinity; C, D: nitrates ( $\mu \mathrm{mol} \mathrm{L}^{-1} \mathrm{~N}-\left(\mathrm{NO}_{3}+\mathrm{NO}_{2}\right)$ ): E, F: chlorophyll a ( $\mu$ mol $\mathrm{L}^{-1}$ ) in $\mathrm{April}_{\text {and June }}$ 1994. The limit of the euphotic zone is indicated by dashed line and sampling levels by dark circles.

mal du panache $\left(1,5 \times 10^{5}\right.$ cellules par litre en surface et $4 \times 10^{5}$ cellules par litre au fond) (figure $5 a$ ), il diminue avec la dilution du panache mais subsiste jusqu' au point distal. On observe un gradient horizontal d'abondance particulièrement prononcé en dessous de la zone éclairée. Les eaux du panache sont également caractérisées par la 
Tableau I. Température $\mathrm{T}\left({ }^{\circ} \mathrm{C}\right)$, cocfficient d'extinction de la lumière $\mathrm{k}\left(\mathrm{m}^{-1}\right)$, concentrations des éléments nutritifs $\left(\mu\right.$ mol $\left.\mathrm{L}^{-1}\right)$, en surface, aux différents points du panache $(B, C, D)$ et au point marin $(A)$.

Table I. Temperature $\mathrm{T}\left({ }^{\circ} \mathrm{C}\right)$, light extinction coefficient $\mathrm{k}\left(\mathrm{m}^{-1}\right)$, nutrient concentrations $\left(\mu \mathrm{mol} \mathrm{L}^{-1}\right)$, in the plume surface water $(\mathrm{B}, \mathrm{C}, \mathrm{D})$ and in the marine surface water (A).

\begin{tabular}{|c|c|c|c|c|c|c|}
\hline Avril 1994 & $\mathbf{T}$ & $\mathbf{k}$ & $\mathrm{NO}_{3}^{-}+\mathrm{NO}_{2}^{-}$ & $\mathrm{NH}_{4}^{+}$ & $\mathrm{Si}(\mathrm{OH})_{4}^{-}$ & $\mathbf{P O}_{4}^{\mathbf{3}}$ \\
\hline Point $D(S=25,1)$ & 10,8 & 0.99 & 115 & 6,0 & 27 & 2,5 \\
\hline Point $\mathrm{C}(\mathrm{S}=28,3)$ & 10,8 & 0,43 & 76 & 2,2 & 16 & 1.7 \\
\hline Point $B(S=32,0)$ & 10,1 & 0.31 & 19 & 0.7 & 6,5 & 1,0 \\
\hline Point $A(S=34,5)$ & 9.1 & 0,18 & 7 & 0.6 & 0,7 & 0,3 \\
\hline \multicolumn{7}{|l|}{ Juin 1994} \\
\hline Point D $(S=24,4)$ & 17,3 & 1,44 & 106 & 9.0 & 20 & 3,6 \\
\hline Point C $(S=29,4)$ & 16,6 & 0,57 & 20 & 0.8 & 4 & 1,2 \\
\hline Point $B(S=32.5)$ & 15,2 & 0,35 & 8 & 0,9 & 0,8 & 0.3 \\
\hline Point $A(S=34,3)$ & 13,6 & 0,25 & 1,3 & 0.5 & 0,6 & 0.3 \\
\hline
\end{tabular}
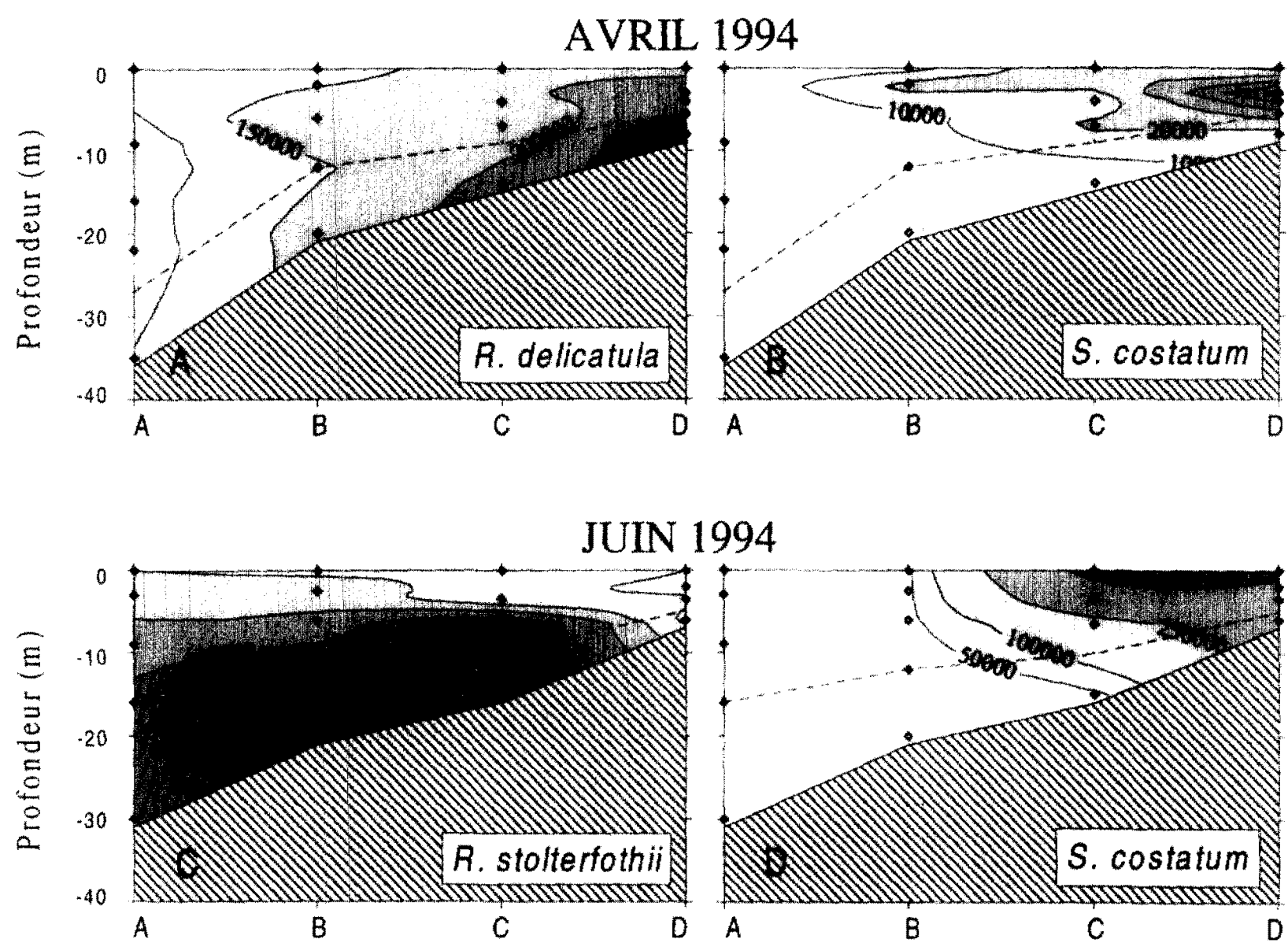

Figure 5. Distribution verticale des espèces microphytoplanctoniques dominantes (cellules $L^{-1}$ ) en baie de Seine, en avril et juin 1994 . A, B : Skeletonema costatum; C : Rhizosolenia delicatuka ; D: Rhizosolenia stolterfothii Les points correspondent aux niveaux de prélèvement; la ligne pointillée représente la limite de la zone euphotique.

Figure 5. Vertical distribution of the dominant microphytoplankton species (cells $\mathrm{L}^{-1}$ ) in April and June 1994. A, B: Skeletonema castatum; C: Rhizosolenia delicatula; D: Rhizosolenia stolterfothii. The limit of the euphotic zone is indicated by dashed line and sampling levels by dark circles. 
présence de diverses espèces de Thalassiosira ( 3 à $7 \times$ $10^{4}$ cellules par litre). Dans les eaux de salinité inférieure à 32, on note la présence de Skeletonema costatum (figure $5 b$ ), notamment au point proximal ( 2 à $6 \times 10^{4}$ cellules par litre). Un nombre relativement important de dinoflagellés ( 4 à $5 \times 10^{4}$ cellules par litre, dans les trois premiers mètres), représentés essentiellement par Protoperidinium trochoüdeum et Heterocapsa triquetra, est observé à ce point. Dans les eaux marines, $R$. delicatula est accompagnée de deux autres espèces du même genre ( $R$. schrubsolei et $R$. fragilissima).

En juin, les concentrations de chlorophylle $a$ restent élevées sur l'ensemble de la zone étudiée avec des valeurs variant de 4,5 à $11 \mu \mathrm{g} \mathrm{L}^{-1}$ dans le panache (figure $4 f$ ). Dans les eaux marines, les teneurs en chlorophylle $a$ varient de 3,5 à $4,5 \mu \mathrm{g} \mathrm{L}^{-1}$. Malgré la stratification, la chlorophylle $a$, aux points proximal et médian du panache, est homogène dans la colonne d'eau. Un maximum de chlorophylle $a\left(8 \mu \mathrm{g} \mathrm{L}^{-1}\right)$ est observé au point distal du panache à la limite et en dessous de la zone euphotique (figure $4 f$ ). La chlorophylle $a$ dans les eaux marines présente, comme au point distal du panache, un maximum $\left(4,5 \mu \mathrm{g} \mathrm{L}^{-1}\right)$ en dessous de la couche éclairée. Le microphytoplancton, dans les eaux du panache, est dominé par les petites cellules de Skeletonema costatum $\left(5 \times 10^{5}\right.$ cellules par litre) (figure $5 d$ ). Cette espèce est accompagnée de Biddulphia aurita au point proximal, et de Rhizosolenia stolterfothii aux autres points. $R$. stolterfothii devient dominante dans les eaux de salinité supérieure à 32 (figure $5 c$ ). Son abondance (> $1,5 \times 10^{5}$ cellules par litre), croissant avec la profondeur, est à l'ori- gine du maximum de chlorophylle $a$ observé dans la couche de fond, au point distal. Les dinoflagellés sont présents sur l'ensemble du panache avec un maximum de densité au point proximal, dans les eaux les plus stratifiées el les plus dessalées $\left(8 \times 10^{4}\right.$ cellules par litre $)$. Ils sont représentés essentiellement par Prorocentrum micans et Scrippsiella faeroense. Dans les eaux marines, la diatomée dominante est Rhizosolenia stolterfothii ; sa distribution verticale est comparable à celle décrite au point distal du panache, avec un maximum d'abondance situé en dessous de la zone euphotique. Cette espèce est accompagnée d'une autre grande diatomée, Rhizosolenia schrubsolei, en faible concentration $\left(2 \times 10^{4}\right.$ cellules par litre) et répartie uniformément dans la colonne d'eau.

\subsubsection{Distribution de la production primaire et des indices de productivité}

En avril, le taux de production de carbone, dans les eaux du panache, varie en surface de $40 \mu \mathrm{g} \mathrm{L}^{-1} \mathrm{~h}^{-1}$ au point proximal à $60 \mu \mathrm{g} \mathrm{L}^{-1} \mathrm{~h}^{-1}$ au point distal (figure $6 a$ ). Dans les eaux marines, la production carbonée de surface est de $14 \mu \mathrm{g} \mathrm{L}^{-1} \mathrm{~h}^{-1}$. Le nombre d'assimilation (quantité maximale de carbone produite par unité de biomasse chlorophyllienne, dans ta colonne d'eau) varie de $7 \mu \mathrm{g}$ $\mu \mathrm{g}^{-1} \mathrm{~h}^{-1}$ aux points proximal et médian du panache à $9,3 \mu \mathrm{g} \mu \mathrm{g}^{-1} \mathrm{~h}^{-1}$ au point distal. 11 est de $3,8 \mu \mathrm{g} \mu \mathrm{g}^{-1} \mathrm{~h}^{-1}$ dans les eaux marines. La production journalière de carbone augmente du point proximal $\left(1,4 \mathrm{~g} \mathrm{~m}^{-2} \mathrm{j}^{-1}\right)$ au point distal du panache $\left(3 \mathrm{~g} \mathrm{~m}^{-2} \mathrm{j}^{-1}\right)$; elle est de $1,5 \mathrm{~g} \mathrm{~m}^{-2} \mathrm{j}^{-1}$ dans les eaux marines (tableat $I I$ ). La productivité des

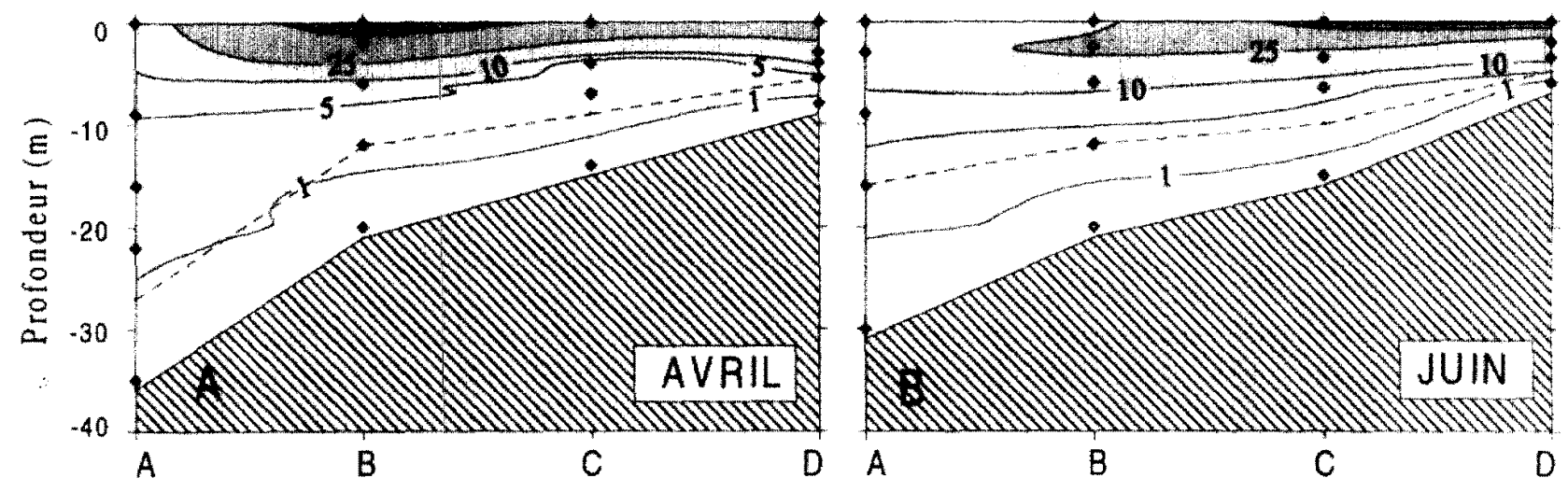

Figure 6. Distribution verticale de la production de carbone $\left(\mu g \mathrm{~L}^{-1} \mathrm{~b}^{-1}\right)$, en baie de Seine en 1994 . A, avril ; B, juin. Les points correspondent aux niveaux de prélèvement ; la ligne pointillée représente la limite de la zone euphotique.

Figure 6. Vertical distribution of carbon productivity $\left(\mu \mathrm{g} \mathrm{L} \mathrm{L}^{-1} \mathrm{~h}^{1}\right.$ ), in the Bay of Seine in 1994. A. April; B, June. The limit of the euphotic zone is indicated by dashed line and sampling levels by dark circles. 
Tableau II. Production de carbone journalière $\left(\mathrm{g} \mathrm{m}^{-2} \mathrm{j}^{-1}\right)$, biomasse chlorophyllienne intégrée dans la couche euphotique ( $\mathrm{mg} \mathrm{m}^{-2}$ ) et productivité de la colonne d'eau (unité de carbone par unité de chlorophylle) ( $m g \mathrm{mg}^{-1} \mathrm{j}^{-\mathrm{f}}$ ) aux différents points du panache (B, $\mathrm{C}$, D) et au point marin (A).

Table II. Daily carbon productivity $\left(\mathrm{g} \mathrm{m}^{-2} \mathrm{~d}^{-1}\right)$, integrated chlorophyll biomass in the photic zone $\left(\mathrm{mg} \mathrm{m}^{-2}\right)$ and carbon productivity per unit of chlorophyll $a\left(\mathrm{mg} \mathrm{mg}^{-1} \mathrm{~d}^{-1}\right)$ in the plume waters $(B, C$. $D)$ and in the marine waters (A)

\begin{tabular}{lccc}
\hline Avril 1994 & Production & Biomasse & Productivité \\
\hline Point D $(S=25,1)$ & 1,4 & 35,4 & 39,5 \\
Point C $(S=28,3)$ & 1,7 & 35,6 & 47,7 \\
Point B $(S=32,0)$ & 3,0 & 74,3 & 40,4 \\
Point A $(S=34,5)$ & 1,5 & 68,3 & 22,0 \\
\hline Juin 1994 & \multicolumn{3}{l}{} \\
\hline Point D $(S=24,4)$ & 1,6 & 39,3 & 40,7 \\
Point C $(S=29,4)$ & 2,75 & 30,5 & 90,1 \\
Point B $(S=32,5)$ & 2,8 & 64,1 & 43,7 \\
Point A $(S=34,3)$ & 2,4 & 55,2 & 43,5 \\
\hline
\end{tabular}

Tableau III. Taux de croissance spécifique $\left(\mu j^{-1}\right)$ du phytoplancton aux différents points du panache $(B, C, D)$ et au point marin $(A)$ en surface et au niveau d'éclairement de $10^{\circ}$ \% de l'éclairement incident de surface.

Table III. Specific growth rate $\left(\mu \mathrm{d}^{-1}\right)$ of phytoplankton in the plume waters $(B, C, D)$ and in the marine waters $(A)$ at surface light and $10 \%$ surface light intensity.

\begin{tabular}{lcc}
\hline Avril 1994 & Surface & $\mathbf{1 0 \%}$ Is \\
\hline Point D $(S=25,1)$ & 0,04 & 0,16 \\
Point C $(S=28,3)$ & 0,23 & 0,03 \\
Point $B(S=32,0)$ & 0,18 & 0,06 \\
Point A $(S=34,5)$ & 0,14 & 0,06 \\
\hline Juin 1994 & & \\
\hline Point D $(S=24,4)$ & 0,07 & 0,46 \\
Point C $(S=29,4)$ & 1,31 & 0,64 \\
Point $B(S=32,5)$ & 0,34 & 0,20 \\
Point A $(S=34,3)$ & 0,33 & 0,19 \\
\hline
\end{tabular}

masses d'eau P/B (production journalière/biomasse chlorophyllienne intégrée dans la couche euphotique, en unité de carbone par unité de Chla) est voisine de $40 \mathrm{mg} \mathrm{mg}^{-1}$ $\mathrm{j}^{-1}$ dans les eaux du panache (tableau II); elle est deux fois plus faible dans les eaux marines $\left(22 \mathrm{mg} \mathrm{mg}^{-1} \mathrm{j}^{-1}\right)$. Le taux de croissance spécifique $(\mu)$ dans les eaux du panache $\left(0,04-0,23 \mathrm{j}^{-1}\right)$ et dans les eaux marines $(0,04$ $0,14 \mathrm{j}^{-1}$ ) est faible (tableau III). II est plus élevé en surface qu'à la profondeur correspondant à $10 \%$ de l'éclaj- rement de surface, à l'exception du point proximal du panache.

En juin, les taux de production de carbone en surface sont trois fois plus élevés aux points proximal et médian $\left(60 \mu \mathrm{g} \mathrm{L} \mathrm{L}^{-1} \mathrm{~h}^{-1}\right)$ qu'au point distal du panache $\left(21 \mu \mathrm{g} \mathrm{L}^{-1}\right.$ $\mathrm{h}^{-1}$ ) et qu'au point marin $\left(18 \mu \mathrm{g} \mathrm{L}^{-1} \mathrm{~h}^{-1}\right.$ ) (figure 6 b). Le nombre d'assimilation varie de $7 \mu \mathrm{g}^{-1} \mathrm{~g}^{-1}$ au point proximal à $10 \mu \mathrm{g} \mathrm{g}^{-1} \mathrm{~h}^{-1}$ au point médian et $5 \mu \mathrm{g} \mu \mathrm{g}^{-1} \mathrm{~h}^{-1}$ au point distal du panache. Dans les eaux marines, le nombre d'assimilation est de $6,6 \mu \mathrm{g} \mu \mathrm{g}^{-1} \mathrm{~h}^{-1}$. À cette époque, la production journalière de carbone est plus faible au point proximal $\left(1,6 \mathrm{~g} \mathrm{~m}^{-2} \mathrm{j}^{-1}\right)$ qu'aux autres points du panache (environ $2,8 \mathrm{~g} \mathrm{~m}^{-2} \mathrm{j}^{-1}$ ) et que dans les eaux marines $\left(2,4 \mathrm{~g} \mathrm{~m}^{-2} \mathrm{j}^{-1}\right)$. La productivité P/B en juin est comparable à celles du panache en avril ( 41 à $\left.44 \mathrm{mg} \mathrm{mg}^{-1} \mathrm{j}^{-1}\right)$ à l'exception du point médian où elle est deux fois plus élevée $\left(90 \mathrm{mg} \mathrm{mg}^{-1} \mathrm{j}^{-1}\right.$ ) (tableau $/$ ). Le taux de croissance spécifique $\mu$, dans les eaux du panache, varie de 0,07 à $1,31 \mathrm{j}^{-1}$ en surface et de 0,2 à $0,64 \mathrm{j}^{-1}$ à la profondeur correspondant à $10 \%$ de l'éclairement de surface (tableau III). Les valeurs les plus élevées sont mesurées au point médian. Dans les eaux marines, le taux de croissance spécifique est de $0,33 \mathrm{j}^{-1}$ en surface et de $0.19 \mathrm{j}^{-1}$ à $10 \% \mathrm{I}_{\mathrm{s}}$. Le taux de croissance spécifique diminue avec la profondeur à l'exception du point proximal du panache. À ce point, le taux de croissance spécifique est nettement plus faible en surface qu'à la profondeur correspondant à $10 \%$ de l'éclairement en surface comme cela a déjà été observé en avril. La faible croissance du phytoplancton en surface pourrait être due à un stress chimique lié aux apports de polluants par les eaux de la Seine plutôt qu"à la dessalure (voir discussion).

\section{DISCUSSION}

La distribution du phytoplancton en baie de Seine, en 1992 et en 1994, est marquée par la dominance persistante des diatomées au printemps et au début de l'été. En 1992, les diatomées représentent de 60 à $80 \%$ du phytoplancton de taille supérieure à 10-15 $\mu \mathrm{m}$; en 1994, des expériences de fractionnement montrent qu'elles constituent de 70 à $95 \%$ de la biomasse chlorophyllienne (résultats non publiés). Ceci n'est pas habituel dans les eaux côtières de la Manche orientale. Dans la frange d'eaux dessalées qui s'étend, le long des côtes françaises, de la baie de Seine au cap Gris-Nez ( fleuve côtier" [7]), la floraison printanière des diatomées est suivie par le développement d'une communauté phytoplanctonique 
dominée par les phytoflagellés [5]. Cette succession est également observée dans de nombreux estuaires, panaches et baies de la bordure orientale des États-Unis d'Amérique $[13,21,24,25,26,27]$ et de l'Atlantique nord européen [12]. Le changement de population est généralement attribué à la réduction des apports en sels nutritifs. En baie de Seine, ces apports diminuent peu pendant la période printanière des deux années étudiées. En 1992, les concentrations en sels nutritifs de la Seine restent élevées jusqu'au début de la période d'étiage (juin) [1]; il en est de même en 1994, comme le montrent les fortes concentrations dans le panache en juin (tableau I). Cet enrichissement permanent peut expliquer le développement continu des diatomées sur l'ensemble de la zone étudiée au cours de la période printanière.

La distribution des densités de diatomées au printemps 1992 (figure 3) montre que leur développement ne s'effectue pas de façon uniforme mais apparaît à des époques différentes selon les secteurs. La floraison débute en avril dans les eaux marines et progresse vers la côte au cours du printemps. Ce phénomène a déjà été observé, notamment, dans la baie d'Hudson [24] et la baie de Chesapeake [13]. Il est lié très vraisemblablement aux variations du niveau moyen d'éclairement dans la colonne d'eau, considéré comme un facteur déterminant pour déclencher le développement printanier du phytoplancton $[4,8,16,23,31,35,38]$. L'énergie lumineuse disponible dans la colonne d'eau dépend de l'énergie solaire et de la turbidité des masses d'eau. En avril 1992, dans la baie de Seine, la turbidité dans les eaux marines $(<1$ NTU) est deux à cing fois plus faible que dans les eaux dessalées (>2 NTU) [17]. De même, en avril 1994, le coefficient d'extinction de la lumière est cinq fois plus faible dans les eaux marines qu'au point proximal du panache (tableau I). Cette faible turbidité favorise la pénétration de la lumière et le développement plus précoce du phytoplancton. Le déclin des populations de diatomées dans les eaux marines n'intervient qu'à l'épuisement du stock hivernal d'éléments nutritifs. Le développement du phytoplancton progresse vers les eaux du panache au cours de la période printanière en raison de l'augmentation de l'éclairement.

Une autre caractéristique de la distribution du phytoplancton en baie de Seine, en 1992 (avril) comme en 1994 (juin), est la prédominance des diatomées de grande taille (Rhizosolenia delicatula, Rhizosolenia stolterfothii) dans les eaux marines et celle des espèces de petite taille (Chaetoceros socialis, Asterionella glacialis, Skeletonema costatum) dans les eaux dessalées. Cette distribu- tion est liée en partie aux variations de salinité. Les petites diatomées présentes dans le panache de la Seine sont des espèces estuariennes à large tolérance haline. Ainsi, S. costatum qui était l'espèce dominante en 1994 se développe en culture avec un taux de croissance spécifique identique dans une gamme de salinité de 10 à 40 [29]. En outre, sa croissance est inchangée qu'elle ait été ou non préadaptée aux changements de salinité du milieu [29]. Cette propriété est un avantage important dans un milieu soumis à de fortes variations de salinité. Par contre, $R$. stolterfothii et $R$. delicatula se développent en milieu naturel dans des eaux de salinité supérieure à 18 et 23 respectivement, et $R$. delicatula n'est abondante qu'à une salinité supérieure ou égale à 30 [34]. La distribution des espèces semble aussi être liée à la structure verticale des masses d'eaux. Les plus fortes densités de $S$. costatum sont en effet observées en surface aux points proximal et médian du panache où la stratification est la plus forte alors que les densités maximales des espèces du genre Rhizosolenia sont généralement relevées dans les masses d'eau homogènes ou peu stratifiees (figures 3 et 5). Une distribution comparable a êté mise en évidence dans le delta du Rhin et de la Meuse [34]. Elle pourrait être due, en partie, à la turbulence nécessaire au maintien en suspension dans la colonne d'eau des grosses diatomées fortement silicifiées [36]. La situation observée en ayril 1994 (figure 5) montre que les diatomées de grande taille ( $R$. delicatula) peuvent être dominantes dans des eaux stratifiées et dessalées. La salinité dans les secteurs du panache qui ont été étudiés n'est probablement pas assez faible pour empêcher leur développement. Elle est, d'une part, toujours supérieure au seuil de tolérance donné par Rijstenbil [34] pour cette espèce et, d'autre part. égale ou supérieure à la salinité optimale d'abondance (30), sauf au point proximal.

Les densités maximales des diatomées de grande taille ne sont pas en général observées dans la couche superficielle éclairée mais au-dessous, dans la zone aphotique. C'est le cas en avril et en juin dans les eaux du panache et également en juin dans les eaux marines (figure 5). De telles concentrations de diatomées ont été observées dans différents systèmes estuariens comme la baie d'Hudson [24], le panache du Mississipi [10], la baie de Chesapeake [13] et le détroit de Georgie [45]. Elles sont généralement attribuées à la sédimentation des cellules. Celle-ci dépend principalement de l'état physiologique et notamment de l'êtat nutritionnel des cellules. La carence en un élément nutritif peut entraîner une forte augmentation de la sédimentation [3]. Dans le panache de la Seine, les teneurs en 
éléments nutritifs sont largement suffisantes, en avril, pour ne pas limiter le développement phytoplanctonique (tableau I). C'est également le cas en juin au point proximal et au point médian ; par contre, au point distal du panache et au point marin, les teneurs en silicates $\left(0,8 \mu \mathrm{mol} \mathrm{L}{ }^{-1}\right)$ et en phosphates $\left(0,2 \mu \mathrm{mol} \mathrm{L} \mathrm{L}^{-1}\right)$ ont fortement chuté par rapport à avril. Dans les eaux marines, en juin, les concentrations en nitrates et en ammonium sont également réduites. Bien que la colonne d'eau ne soit pas épuisée, il est possible que ces faibles concentrations de sels nutritifs soient limitantes pour la croissance de R. stolterfothii. À cette époque, les teneurs en nitrates et en ammonium permettent de satisfaire seulement la moitié de la capacité maximale d'absorption de l'azote par le phytoplancton [20] indiquant ainsi une limitation en cet élément. Ces conditions accentueraient le taux de sédimentation des populations de diatomées. Dans le panache de la Seine, le processus de sédimentation n'affecte que les diatomées de grande taille. Ceci peut être lié à la période d'observation qui correspondrait, dans l'ordre des successions phytoplanctoniques, au déclin de ces populations; mais ce phénomène peut être également attribué à la stratification des eaux dessalées. La turbulence serait insuffisante pour maintenir les cellules en suspension (voir plus haut) ; toutefois, la stratification paraît trop faible pour constituer une barrière physique empêchant la sédimentation des diatomées de grande taille. Les maximums de chlorophylle au-dessous de la zone euphotique peuvent être provoqués par des phénomènes d'advection des eaux marines sous les eaux dessalées comme dans la baie de Chesapeake [27] et le détroit de Géorgie [45]. D'après Malone et al. [27], le phytoplancton se développe dans les eaux dessalées de surface pendant leur transit. Le processus de sédimentation entraîne la biomasse au large vers la couche de fond. Les eaux de cette dernière sont ensuite advectées vers la côte en dessous des eaux du panache, induisant ainsi la formation de maximum de biomasse dans la couche aphotique. En baie de Seine, les simulations de Le Hir et al. [19] ont montré que par vent de secteur ouest (cas des deux campagnes), un contre-courant de fond pouvait se former dans la paléovallée de la Seine entrainant les particules en suspension vers la côte [2]. Ce mécanisme pourrait expliquer en partie la distribution horizontale et verticale de $R$. delicatula en avril et de $R$, stolterfothii, en juin (figure 5).

La production primaire présente, en avril, des valeurs nettement plus élevées dans les eaux du panache que dans les eaux marines (figure $6 a$ ). Ces valeurs augmentent du point proximal au point distal du panache en raison inverse du coefficient d'extinction de la lumière (tableau $I$ ). Par contre, les valeurs de production primaire intégrées dans la zone euphotique sont, à l'exception du point distal, comparables dans les eaux marines et dans les eaux du panache (tableau $I$ ). La productivité des masses d'eau est identique à tous les points du panache, correspondant à des communautés phytoplanctoniques similaires dominées par $R$. delicatula. La forte augmentation de la production primaire au point distal du panache pourrait être due à une meilleure pénétration de la lumière dans la colonne d'eau. Dans les eaux marines, la productivité du phytoplancton est deux fois plus faible que dans les eaux du panache (tableau $I$ ); ceci ne semble pas lié aux teneurs en sels nutritifs qui sont suffisamment álevées, à cette époque de l'année, pour ne pas limiter le développement phytoplanctonique. La productivité réduite du phytoplancton peut être liée au brassage des eaux qui entraîne périodiquement le phytoplancton dans la zone non éclairée. Les populations phytoplanctoniques s'adaptent dans ce cas à un éclairement moyen [11], ce qui se traduit par une diminution de la productivité (et par conséquent une diminution du taux de croissance). Elle est compensée dans les eaux marines par une utilisation du carbone sur une plus grande profondeur dans la colonne d'eau; ceci conduit à une production journalière de carbone aussi élevée dans les eaux marines que dans les eaux enrichies du panache.

En juin, les taux de production, en surface, sont trois fois plus élevés aux points proximal et médian du panache qu'au point distal et qu'au point marin (figure $6 b$ ). À cette époque, les variations de la production semblent être liées à la composition des communautés phytoplanctoniques dominées soit par S. costatum (points proximal et médian) soit par $R$. stolterfothii (point distal et point marin). La production primaire intégrée sur l'ensemble de la zone euphotique est identique dans les eaux marines et dans les eaux du panache, à l'exception du point proximal. Contrairement à la situation d'avril, la productivité des eaux marines est aussi élevée que celle des eaux du panache (tableau $(I)$. La distribution verticale de la chlorophylle n'est pas homogène en raison des phénomènes de sédimentation et/ou d'advection décrits plus haut. De ce double point de vue, point marin et point distal du panache ne semblent pas très différents. Les indices biologiques montrent également une grande similitude entre ces deux points : tous les paramètres biologiques mesurés (nombre d'assimilation du carbone, production journalière, productivité des masses d'eau, taux de croissance spécifique) sont, respectivement, du même ordre de grandeur. À ces deux points, la bonne pénétration de la lumière induit des 
Tableau IV. Gamme des valeurs de production de carbone $\left(\mathrm{g} \mathrm{m}^{-2} \mathrm{j}^{-1}\right)$ et de productivité des masses d'eau (production de carbone par unité de biomasse chlorophyllienne intégrée dans la couche euphotique en $\mathrm{mg} \mathrm{mg}^{-1} \mathrm{j}^{-1}$ ) dans différents systèmes estuariens de l'Atlantique nord.

Table IV. Value range of daily carbon productivity $\left(\mathrm{g} \mathrm{m}^{-2} \mathrm{~d}^{-1}\right)$ and carbon productivity per unit photic zone chlorophyll $a\left(\mathrm{mg} \mathrm{mg}^{-1} \mathrm{~d}^{-1}\right)$ in various North Atlantic estuary systems.

\begin{tabular}{|c|c|c|c|c|c|}
\hline Lien & Salinité & 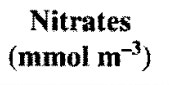 & $\begin{array}{l}\text { Production } \\
\left(\mathrm{g} \mathrm{m}^{-2} \mathrm{j}^{-1}\right)\end{array}$ & $\begin{array}{l}\text { Productivitế } \\
\left(\mathbf{m g ~} \mathbf{m g}^{-1} \mathbf{j}^{-1}\right)\end{array}$ & Références \\
\hline Baie d'Hudson, NY, USA & $28 \cdot 32$ & $1-14$ & $0,1-0,6$ & $40-65$ & Malone [24] \\
\hline Baie de Peconic, NY, USA & $24-29$ & $0,05-39,5$ & $0-2,1$ & $0-110$ & Bruno et al. [6] \\
\hline Great South Bay, NY, USA & $22-30$ & $<2$ & $0,3-2,9$ & - & Lively et al. [2I] \\
\hline Estuaire de la Delaware, DE, USA & - & - & $0,01+3,2$ & - & Pennock et Sharp [31] \\
\hline Panache du Mississipi, MS, USA & $21-34$ & $<0,02-23,6$ & $0,12-3,0$ & - & Redalje et al. $[33]$ \\
\hline Baie de Southampton, G.B. & $26-35$ & $2-65$ & $0,1-3,5$ & - & Iriarte et Purdie \15] \\
\hline Baie de Seine, France & $25-34$ & $1,3-115$ & $1,4-3,0$ & $22 \cdot 90$ & cette étude \\
\hline
\end{tabular}

valeurs élevées de production primaire. Au point médian, bien que la biomasse chlorophyllienne de la zone euphotique soit deux fois plus faible, la production primaire est aussi élevée qu'aux points précités, en raison de la forte productivité du phytoplancton. Cette productivité est associée à la population de $S$. costatum qui semble bénéficier

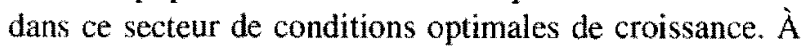
ce point, en effet, tous les paramètres biologiques atteignent les plus fortes valeurs mesurées au cours de l'étude. Stratification, salinité, lumière, éléments nutritifs abondants induisent un taux de croissance spécifique $\left(\mu=1,3 \mathrm{j}^{-1}\right)$ identique au taux de croissance spécifique maximum de cette espèce en culture [29].

La production primaire et la productivité mesurées en baie de Seine sont comparables aux valeurs habituellement citées pour les systèmes estuariens (tableau IV). Les valeurs maximales sont proches de celles mesurées, pour une gamme de salinité comparable, par Malone [24] dans la baie d'Hudson et par Bruno et al. [6], dans la baie de Peconic ; elles sont proches également des valeurs mesurées dans le panache du Mississipi [33]. La production primaire semble donc varier relativement peu d'un système à un autre alors que l'enrichissement en éléments nutritifs présente d'importantes différences, comme le montre la gamme de variations des teneurs en nitrates (tableau $\mathrm{IV}$ ). La similitude des valeurs de production suggère que le développement du phytoplancton est davantage contrôlé par le faible niveau d'éclairement prévalant dans les eaux turbides que par la disponibilité d'éléments nutritifs.

\section{CONCLUSIONS}

Le système du panache de la Seine se distingue des autres systèmes décrits dans la littérature par la présence perrnanente de diatomées du début du printemps au début de l'été. Les eaux du panache sont caractérisées par la dominance d'espèces de petite taille alors que les diatomées de grande taille se développent préférentiellement dans les eaux de salinité supérieures à 32. Les fortes concentrations de chlorophylle observées en dessous de la zone euphotique résultent de la sédimentation et/ou de l'advection des diatomées de grande taille. De façon générale, le niveau de production phytoplanctonique en baie de Seine semble être davantage contrôlé par la lumière que par la concentration en éléments nutritifs.

\section{Remerciements}

Ce travail est une contribution au Programme national d'océanographie côtière Ifremer-CNRS/INSU (thème «eutrophisation $»)$.

\section{RÉFÉRENCES}

[1] Aminot A., Guillaud J.F, Andrieux-Loyer F, Kérouel R., Cann P.. Apports de nutriments et développement phytoplanctonique en baie de Seine, Oceanol. Acta 21 (6) (1998), ce volume.
[2] Avoine J., Crevel C., Influence des apports fluviatiles en baie de Seine, in : La baie de Seine (Greco-Manche), Ifremer (ed.), Actes de Colloques $n^{\circ} 4$, univ. Caen, 24-26 avril 1985 (1986) $125-134$. 
[3] Bienfang P.K, Harrison P,J., Quarmby L.M., Sinking rate response to depletion of nitrate, phosphate and silicate in four marine diatoms, Mar. Biol. 67 (1982) 295-302.

[4] Boynton W.R., Kemp W.M., Keele C.W., A comparative analysis of nutrients and other factors influencing estuarine phytoplankton production, in: Kennedy V.S. (éd.), Estuarine comparaisons, Academic Press. Londres, 1982, 69-90.

[5] Brunet C., Brylinski J.M., Bodineau L., Thoumelin G., Bentley D. Hilde D., Phytoplankton dynamics during the spring bloom in the south eastern English Channel, Estuar. Coast. Shelf Sci. 43 (1996) $469-483$.

[6] Bruno S.F., Staker R.D. Shama G.M., Dynamics of phytoplankton productivity in the Peconic Bay estuary, Long Island. Estuar, Coast. Mar. Sci. 10 (1980) 247-263.

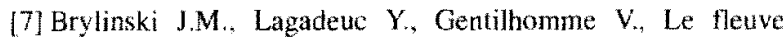
côtier, un phénomène hydrologique importan en Manche orientale (exemple du Pas-de-Calais), Oceanol. Acta 11 (1991) 197-203.

[8] Cole B.E, Cloem J.E., Significance of biomass and light avaibility to phytoplankton productivity in San Franciseo Bay, Mar. Ecol. Prog. Ser. 17 (1984) 15-24.

[9] Crével L., Contribution à l'étude de la dynamique sếdimentaire dans la partie nord-orientale de la baje de Seine, thèse de $3^{c}$ cycle, univ. Caen (1983) $164 \mathrm{p}$.

[10] Dortch Q. Whitledge T.E, Does nitrogen or silicon limit phytoplankton production in the Mississipi river plume and nearby regions? Cont. Shelf Res. 12 (1992) 1293-1309.

[11) Falkowski P.G., Light-shade adaptation and vertical mixing of marine phytoplankton, A comparative field study, J. Mar. Res. 41 (1983) $215-237$.

[12] Fransz H.G., Effects of fresh water inflow on the distribution. composition and production of plankton in the Dutch coastal waters of the North Sea, in: Kreslet S. (éd.), The role of freshwater outflow in coastal marine ecosystems, Springer-Verlag. Heidelberg, 1986, 241-249.

[13] Glibert P., Conley D.J., Fisher T..R, Harding L.W. Jr, Malone T.C. Dynamics of the 1990 winter/spring bloom in Chesapeake Bay, Mar. Ecol. Prog. Ser. 122 (1995) 27-43.

[14] Guillaud J.F., Monbet Y., Romana A., Interprétation des résultats du Réseau national d'observation de la qualité du milieu marin, première phase, point d'appui : baie de Seine, Rapport Cnexo, féx: 1978 (1978) 85 p.

[15] Iriarte A., Purdie D.A., Size distribution of chlorophyll a biomass and primary production in a temperate estuary (Southampton Water): the contribution of photosynthetic picoplankton, Mar. Ecol. Prog. Ser. 115 (1994) 283-297.

[16] Joint I.R., Pomroy A.J., Primary production in a turbid estuary, Estuar. coast Shelf Sci. 13 (1981) 303-316.

[17] Kérouel R., Aminot A., Cann P.. Les nutriments en baie de Seine, in: Eutrophisation des écosystèmes côtiers et cycles biogéochimiques de l'azote et du phosphore, Programme national d'océanographie côtière, CNRS, Rapport de l'année $1993(1994) 9-66$.

[18] Koroleff F., Direct determination of ammonia in natural waters as indophenol blue, in: Information on techniques and methods in seawater analysis, Rapp. P-v Réun. Cons. perm.int. Explor. Mer. 3 (1970) 19-22.

[19] Le Hir P.. Salomon J.C, Le Provost C., Chabert d'Hières G., Mauvais J,L., Approche de la circulation résiduelle en baie de Seine, in: Ifremer (éd), La baie de Seine (Greco-Manche), Actes de Colloques $n^{\circ} 4$, univ. Caen, 24-26 avril 1985 (1986) p. $63-72$.

[20] L'Helguen S., Le Corre P., Étude des flux de nitrates et d'ammonium dans le phytoplancton du panache de la Seine, Rapport convention Ifremer. Programme national d'océanographie côtière (thème eutrophisation) (1995) $17 \mathrm{p}$.

[21] Lively J.S., Kaufman Z., Carpenter E.J., Phytoplankton ecology of a barrier islland estuary: Great South Bay, New York, Estuar. coast. Shelf Sci. 16(1983) 51-68.

[22] Lorenzen C.J., A method for the continuous measurement of in vivo chlorophyll concentration, Deep-Sea Res. 13 (1966) $223-227$.

[23] Lohrenz S.E., Dagg M.J., Whitledge T.E., Enhanced primary production at the plume/oceanic interface of the Mississipi River, Cont. Shelf Res. 10 (1990) 639664.

[24] Malone T.C., Phytoplankton productivity in the apex of the New York Bight: environmental regulation of productivity/ chlorophyll a. Am. Soc. Limnol. Oceanogr, Spec. Symp. 2 (1976) $260-272$.

[25] Malone T.C., Environmental regulation of phytoplankton productivity in the lower Hudson estuary, Estuar. Coast. Mar. Sci. $5(1977) 157-171$.

[26] Malone T.C., Chervin M.B., The production and fate of phytoplankton size fraction in the plume of the Hudson river, New York Bight, Limnol. Oceanogr. 24 (1977) 683-696.

[27] Malone T.C., Crocker L.H., Pike S.E., Wendler B.W., Influences of river flow on the dynamics of phytoplankton production in a partially stratified estuary, Mar. Ecol. Prog. Ser. 48 (1988) $235-249$.

[28] Ménesguen A., Guillaud J.F., Aminot A., Hoch T., Modelling the eutrophication process in a river plume: the Seine case study (France), Ophelia 42 (1995) 205-225.

[29] Patsche E., The influence of salinity on the growth of some planktonic diatoms from brackish water, Nor. J. Bot. 22 (1975) $209-215$

130] Paulmier G., Joly J.P., Les phénomènes d'eaux colorées en baie de Seine, in : Ifremer (éd.), La baie de Seine (Greco-Manche), Actes de Colloques $n^{\circ} 4$, univ. Caen, 24-26 avril 1985 (1986) $477-483$.

[31] Pennock J.R., Sharp J.H., Phytoplankton production in the Delaware Estuary: temporal and spatial variability, Mar. Ecol. Prog. Ser 34 (1986) 143-155.

[32] Redalje D.G., Laws E.A., A new method for estimating phytoplankton growth rates and carbon biomass, Mar. Biol. 62 (1) (1981) $73-79$.

[33] Redalje D.G., Lohrenz S.E., Fahnenstiel G.L., The relationship between primary production and the vertical export of particulate organic matter in a river-impacted coastal ecosys. tem, Estuaries 17 (1994) 829-838. 
[34] Rijstenbil J.W., Phytoplankton composition of stagnant and tidal ecosystems in relation to salinity, nutrients, light and turbulence, Neth. J. Sea Res. 21 (1987) 113-123.

[35] Riley G.A., Phytoplankton of the North central Sargasso Sea, Limnol. Oceanogr. 2 (1957) 252-270.

[36] Smayda T.J., The phytoplankton of estuaries, in: Ketchum B.H. (ed.), Estuaries and enclosed seas, Ecosystems of the world, 26 (1983) 65-102.

[37] Steemann Nielsen E. The use of radioactive carbon $\left({ }^{14} \mathrm{C}\right.$ ) for measuring organic production in the sea, J. Cons. Int. Explor. Mer 17 (1952) 117-140.

[38] Townsend D.W., Cammen L.M., Holligan P.M., Campbell D.E., Pettigrew N.R., Causes and consequences in the timing of spring blooms, Deep-Sea Res. 41 (1994) 747-764.

[39] Tréguer P., Le Corre P., Manuel d'analyse des sels nutritifs dans l'eau de mer (Utilisation de l'Autoanalyser II - Technicon), $2^{\mathrm{e}}$ edition, univ. Bretagne Occidentale (1975) $110 \mathrm{p}$.

[40] Utermölh H., Neue Wege in der quantitativen Erfassung des Planktons (mit besonserer Berucksichtigung des Ultraplank- tons), Verh. int. Ver. theor. angew. Limnol. 5 (2) (1931) $567-$ 596.

[41] Videau $\mathrm{C}$., Mesure de la croissance du phytoplancton dans la zone du front thermique d'Ouessant en période estivale, $C . R$. Acad. Sci. Paris, 306 (1988) 51-56.

[42] Videau C., Sournia A., Prieur L., Fiala M., Phytoplankton and primary production characteristics at selected sites in the geostrophic Almeria-Oran front (SW Mediteranean Sea), J. Mar. Systems 5 (1994) 235-250.

[43] Welschmeyer N.A., Lorenzen C.J., Carbon-14 labelling of phytoplankton carbon and chlorophyll a carbon: determination of specific growth rate, Limnol. Oceanogr. 29 (1) (1984) 135 145 ,

[44] Yentsch C.S., Menzel D.W., A method for determination of phytoplankton chlorophyll and phaeophytin by fluorescence, Deep-Sea Res. 10 (1963) 221-231.

[45] Yin K., Harrison P.J., Pond S. Beamish R.J., Entrainment of nitrate in the Fraser River estuary and its biological implications, 1. Effects of the salt wedge, Estuar. Coast. Shelf Sci. 40 (1995) $505-528$. 